\title{
Medien und Governance
}

\subsection{Governance - ein interdisziplinäres, komplexes Untersuchungsfeld}

Medien bzw. Kommunikation lassen sich seit jeher nicht und heute noch weniger territorial oder gar national begrenzen; sie sind in ihren Prozessen und Inhalten aterritorial sowie transnational. Allenfalls ihre gesetzliche und administrative Organisation wurde und wird vorwiegend von den im 18. Jahrhundert gebildeten Nationalstaaten bestritten. Schon die elektronischen Medien, Hörfunk und Fernsehen, überwanden - zumal dank moderner Übertragungskanäle und -netze wie Kabel und Satellit - die ehedem durch Funkwellen, mithin terrestrisch begrenzten Reichweiten. Ihre Produktion erfolgt daher durch mehr und mehr transnational oder gar global agierende, breit aufgestellte und verflochtene Konzerne, die inzwischen meist multi- und crossmedial ausgerichtet sind und sämtliche medialen Kanäle bedienen. Vollends sprengt das Internet alle territorialen und nationalen Grenzen und Einordnungen.

Wiewohl noch nicht hinlänglich, zumal weltweit einheitlich angewendet (Wessler und Brüggemann 2012, 2ff), scheinen sich inzwischen - mindestens für die deutsche Forschung - folgende Begriffsklärungen einzubürgern: International meint Beziehungen und Interaktionen zwischen den Nationen bzw. Staaten. Transnational bezieht sich auf jene, die sich nicht nur zwischen den Nationen und Staaten als ganze entwickeln, sondern auch innerhalb, jenseits dieser oder gar quer zu ihnen, mithin unter diversen Gruppierungen, Organisationen und Akteuren, also auch Medien, die eine moderne Nation bzw. einen modernen Staat konstituieren und auszeichnen. Diese Beziehungen und Interaktionen kümmern sich nicht mehr um nationale 
Grenzen; sie überwinden sie, ob absichtlich oder systembedingt. Denn mit den Transformationen des Nationalstaates entwickeln sich Funktionen und Prozesse jenseits der oder quer zu den traditionellen Formen des Regierens, besonders durch zivilgesellschaftliche Engagements und Aufgabenübernahmen, so dass die überkommenen Bestimmungen und Funktionen des Staates erodieren oder auf andere Instanzen übertragen werden (Benz u. a. 2007). Solche Prozesse vollziehen sich auf allen politischen Ebenen, von der lokalen Bürgerbewegung bis hin $\mathrm{zu}$ international und global agierenden Nichtregierungsorganisationen, so genannten INGOs (International Nongovernmental Organizations). Schließlich umreißt supranational die Beziehungen und Interaktionen, die sich oberhalb der Nationen und Staaten vollziehen, den Staaten einen Regelungsrahmen vorgeben, etwa in der Welthandelsorganisation (WTO) oder der Europäischen Union (EU).

Infolge der wachsenden Globalisierung, also der fortschreitenden Verflechtung von wirtschaftlichen und finanziellen Beziehungen der Staaten und Gesellschaften weltweit, haben sich mithin die politischen und hoheitlichen Funktionen des Staates gewandelt, womöglich abgeschwächt oder auch zum Teil auf andere Akteure (stakeholders) verlagert, wie in Kap. 5 eingehender dargestellt wird. Die begriffliche und analytische Folge ist, dass neue, treffendere Termini und - mit ihnen verbunden bzw. ihren vorausgehend - angemessenere Analyseansätze erprobt werden wie der Begriff der Global Governance, der für viele gesellschaftliche und politische Aktionsfelder, aber auch für Medien (Media Governance) (Donges und Puppis 2010; Kleinsteuber und Nehls 2011) angewendet wird.

Dennoch hapert es trotz all dieser Entwicklungen noch an adäquaten interdisziplinären Forschungen, die zum einen die faktisch verflochtenen, komplexen Phänomene analytisch in den Blick nehmen und zum anderen über die speziellen eigenen Fachgrenzen hinaus mit ebenfalls befassten Wissenschaftsdisziplinen integrativ kooperieren. Für Media bzw. Internet Governance kommen primär die Politikwissenschaft mit ihrem Schwerpunkt der inter- bzw. transnationalen Beziehungen sowie eine ebenso ausgerichtete Kommunikationswissenschaft in Frage. Beide Disziplinen - so eine jüngere vergleichende Übersicht (Tenscher und Viehrig 2010, 8) - haben die globalisierenden und sich verzahnenden Entwicklungen zumal hinsichtlich Kommunikation und Medien noch nicht hinreichend berücksichtigt und in theoretische Konzepte umgesetzt; vielmehr sind sie mehrheitlich noch eher auf nationalstaatliche oder internationale Akteure bzw. Systeme (wie etwa die EU) fokussiert: „Eine originär internationale Perspektive auf grenzüberschreitende Kommunikationsprozesse im supra- und internationalen Bereich, die trans- und supranationale Akteuren, intergouvernementalen und Nichtregierungsorganisationen (NGOs) besondere Aufmerksamkeit schenken würde, ist erst in Ansätze zu beobachten [...]“" (Ebd., 9). 
Dabei gilt es zu unterscheiden, ob eine eher international vergleichende oder eine abstrakt ansetzende globale Perspektive gewählt wird (Wessler und Brüggemann 2012, VIff; vgl. Meckel 2001; Hepp 2004; Hepp u. a. 2005; Hartmann 2006; Kübler 2011). Denn der Systemvergleich wird schon länger in beiden Disziplinen, aber weitgehend unabhängig voneinander betrieben (Esser und Pfetsch 2003; Thomaß 2007; Melischek u. a. 2008). Außerdem haben sich unterschiedliche Schwerpunkte und - entsprechend - Lücken der wissenschaftlichen Aufmerksamkeit eingebürgert, was etwa Schwellenländer und bestimmte Kontinente angeht (zuletzt HansBredow-Institut 2009). Zur Tradition der internationalen Perspektive lassen sich auch Darstellungen internationaler supranationaler Organisationen und Prozesse zählen, wie sie vor allem innerhalb Europas gegeben sind (z. B. Holtz-Bacha 2006, 2011). Für genuin globale Phänomene und Entwicklungen werden - jenseits einer nur feuilletonistischen Terminologie (Luhmann 1975; Bolz 2001) - analytisch brauchbare und ergiebige Kategorien und Theorien noch gesucht bzw. erprobt. Diese Einschränkung gilt auch für diese Ausführungen (Abb. 2.1).

Immerhin scheinen beide Disziplinen in den beiden letzten Jahrzehnten bestrebt zu sein, diese Lücken und Desiderate aufzuarbeiten, in der Kommunikationswissenschaft mindestens durch etliche besagte Reader und Lehrbücher (siehe oben). Dabei ändert oder weitet sich der Blickwinkel entsprechend den zeitgenössischen

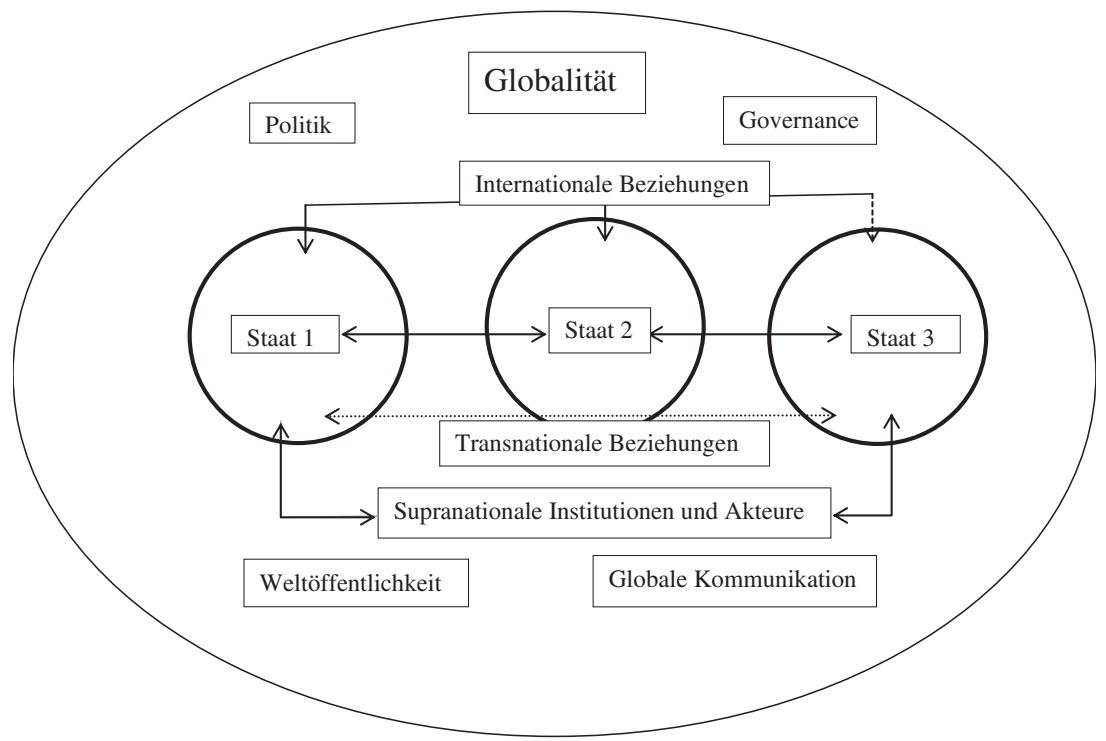

Abb. 2.1 ,Welt' und ,Weltkommunikation`- Analyseansätze und -begriffe (eigene Darstellung) 
Diskussionen und Vokabeln: zum einen weg von der internationalen Kommunikation und Öffentlichkeit zwischen den Staaten hin zur globalen, wie sie sich durch international agierende Medien- und Internetkonzerne und ihre transkulturellen Produkte ergibt bzw. darstellt, aber auch zum zweiten weg von einer irgendwie national konstituierten Kommunikation hin zu diversen transkulturellen Kommunikationsprozessen. In wieweit es sich dabei nur um eine verbale Transformation handelt oder tatsächlich auch andere, größere und komplexere Sachverhalte in den analytischen Blick genommen werden, werden die folgenden Kapitel zu klären versuchen.

Die Politikwissenschaft ist im Vergleich zur Kommunikationswissenschaft schon länger und breit international aufgestellt, zumal durch die angloamerikanische Forschung (Marcinkowski und Pfetsch 2009). Eine Beschränkung nur auf die deutsche Forschung ist daher unzulänglich; damit sind aber zunächst nur Tempo und Intensität der Wissenschaftsdiffusion markiert, nicht aber der mögliche Erkenntnisstand. Denn die hiesige Politikwissenschaft hat (politische) Kommunikation und Öffentlichkeit, seien sie national oder seien sie international und global ausgerichtet, mehrheitlich nur marginal beachtet. Erst allmählich gewinnt sie über die internationale Kommunikation, über Fragen nach Generierung und Konstituenten transnationaler Öffentlichkeiten die erweiterte Perspektive. Jeweils werden die Disziplinen zu sondieren und zu begründen haben, was ihre spezifischen Erkenntnis- und Forschungsanteile sind bzw. sein sollen, um komplexen, wohl auch diffusen Phänomenen - wie Kommunikation, kollektive Bewusstseins- und Meinungsbildung, Öffentlichkeit, Diffusion von Inhalten und Symbolen nun einmal sind - analytisch beizukommen.

Dabei gilt es zunächst zu prüfen, ob eingeführte, traditionelle Begriffe und Kategorien noch zeitgemäß und vor allem sachlich adäquat sind. Dieser Aufgabe stellt sich auch diese Einführung. Mit dem inzwischen viel gebrauchten Terminus Governance könnte sich zudem eine ergiebige und anregende Schnittstelle ergeben, an deren Entwicklung und Differenzierung beide Disziplinen aus jeweils ihren Perspektiven arbeiten und für deren besagte Komplexität sie angemessene Ergebnisse hervorbringen können. Allerdings ist Governance keineswegs eindeutig und präzise zu definieren. Man kann diesen Umstand beklagen, wie es viele tun; man kann darin aber auch eine Erkenntnischance erblicken, um die nach wie vor noch sondierende Erkenntnisarbeit und Theoriefindung offen, unvoreingenommen und möglichst kreativ fortzusetzen und damit den Phänomenen wie Tendenzen näherzukommen (Schuppert 2006, 2011). In jedem Fall bleibt er für etliche Disziplinen - Rechts- Politik-, Wirtschafts- und Kommunikationswissenschaft - anschlussfähig und kann als „Brückenbegriff“ in vielerlei Richtungen (Kleinsteuber 2011, 67ff) fungieren. 
In einem ersten, noch groben Zugriff umfasst Governance folgende Dimensionen; ihre Herleitung und Einordnung erfolgen im nächsten Kapitel:

1. Governance wird sowohl als analytisch-theoretisches Konstrukt verwendet, um vorgeblich neue Sachverhalte, deren Substanz und Transformationen noch nicht hinreichend geklärt sind, möglichst offen, für diverse Disziplinen zu umreißen. Dabei ist zu fragen: welche Sachverhalte sind gemeint? Wo sind die Grenzen bzw. Unterscheidungskriterien zu anderen?

2. Governance dient auch als Bezeichnung für tendenziell noch nicht hinlänglich empirisch erfasste Dynamiken und Veränderungen realer Prozesse und Sachverhalte. Dann wird von Governance-Strukturen bzw. -institutionen, -prozessen bzw. -mechanismen und -akteuren gesprochen. Governance ist damit ein Regulierungssystem, dessen mögliche reale Transformationen empirisch erfasst werden müssen (Donges und Puppis 2007, 13).

3. Governance wird als normativer, wenn nicht gar als aktionaler Begriff gebraucht, mithin als Anspruch, Anforderung oder gar Handlungsbedarf (Empfehlungen), um reale Veränderungen zu legitimieren oder nicht $\mathrm{zu}$ legitimieren oder Szenarios zu entwickeln, wie (noch) nicht regulierte oder regulierbare Handlungsfelder zu gestalten sind. Diese Sichtweise mündet in Normen und Ansprüche von "Good Governance“. Sie signalisieren Nähe $\mathrm{zu}$ ethischen Fragestellungen und Zieldiskussionen vornehmlich unter dem Eindruck der Ablösung überholter Ansätze und Legitimationen.

\subsection{Governance - als politikwissenschaftliche Kategorie}

Der Begriff Governance hat seit den 1980er Jahren in der politik- und sozialwissenschaftlichen Diskussion schnell Karriere gemacht, ist aber dennoch unklar und diffus geblieben, vor allem deswegen, weil ganz unterschiedliche Entwicklungen und Problemstellungen mit ihm belegt wurden (vgl. Van Keersbergen 2004). Er ist nicht mit dem Begriff Regierung, auch nicht mit Regierungsführung zu verwechseln, obwohl natürlich zwischen allen drei Termini starke Schnittmengen existieren. Der Begriff Governance beinhaltet, dass heute nicht mehr nur Regierungen allein allgemeinverbindliche Regelungen planen, beschließen und umsetzen, sondern auch andere Akteure daran beteiligt sind. Dadurch sollen - so die Erwartungen und Hoffnungen jener, die dieses Konzept propagieren - diese Regelungen effektiver greifen, sich ihre gesellschaftliche Legitimität erhöhen und damit auch die Chance 
wachsen, dass diese Regelungen beachtet werden. Diese anderen Akteure sind je nach Breite der Definition Unternehmen, Unternehmensverbände, Vertretungen der Arbeitnehmer, der freien Berufe, Verbände, Nichtregierungsorganisationen, Vertreter sozialer Bewegungen und der Wissenschaft, kurzum: die gesamte traditionelle und neue „Zivilgesellschaft“. Für viele Beobachter des neuzeitlichen Regierens ist es eine ausgemachte Tatsache, dass deren Beteiligung an gesamtgesellschaftlich bindenden Entscheidungen die Qualität des kollektiven Entscheidens und Handelns verbessert, mithin "Good Governance“ fördert. Dies kann man als „Gutes Regierungshandeln“ übersetzen, läuft dabei aber Gefahr zu übersehen, dass daran nicht nur Regierungen beteiligt sind. "Good Governance" meint die effektive Beteiligung möglichst aller relevanten gesellschaftlichen Gruppen an der Beratung über kollektive Programme, Entscheidungen und deren Durchführung, die Rechenschaftspflichtigkeit der mit der Entscheidungsumsetzung betrauten politischen Akteure gegenüber den Bürgern und die Transparenz staatlichen Handelns. Forderungen nach Verbesserung der so verstandenen Regierungsführung werden deshalb vor allem an die Adresse jener Staaten gerichtet, die sich durch entsprechende Defizite auszeichnen. Im Begriff Governance schwingt also oft besagte normative Vorstellung mit, nach welchen Verfahren und nach welchen Normen kollektive Entscheidungen getroffen werden sollen.

Neuerdings erfreut sich der Begriff Governance deshalb einer hohen Popularität, weil die meisten Autoren davon ausgehen, dass spätestens seit den 1970er Jahren ein sukzessiver Formwandel des Staates stattfindet, der seinen Charakter von dem eines „Herrschaftsmonopolisten“ zum „Herrschaftsmanager" wandelt (Genschel und Zangl 2008, 431). Der Staat kann nicht mehr erfolgreich das Monopol über politische Entscheidungen beanspruchen, vielmehr gibt er das Entscheidungsmonopol zunehmend an inter- und supranationale Institutionen, aber auch an nationale nicht-staatliche Akteure ab. Deren Bemühungen ergänzt und koordiniert er zwar noch, bestimmt sie aber nicht mehr vollständig. Dies geschieht freiwillig, weil der Staat nicht mehr alle erforderlichen Leistungen erbringen kann oder will und weil die Herrschaftsbeteiligung nicht-staatlicher Akteure Vorteile in Bezug auf die Effektivität und die Legitimität kollektiver Regelungen erhöht. Natürlich geschieht dies nicht in allen Herrschaftsbereichen gleichermaßen, vielmehr verständlicherweise in jenen Bereichen am wenigsten, bei denen der harte Kern staatlicher Souveränität (etwa die Garantie von Sicherheit nach außen oder das Steuermonopol) betroffen ist. Der Vorgang der ,Entstaatlichung' vollzieht sich nicht gleichzeitig und mit gleicher Breite und Tiefe in allen Nationalstaaten. Vor allem die Regierungen von Entwicklungsländern, die ihre Unabhängigkeit mühsam und spät errungen haben, sind darauf bedacht, ihre Herrschaftsgewalt und die Souveränität der von ihnen geführten Staaten zu wahren. Entstaatlichung' ist also weder flächendeckend 
noch universal, sondern ein Produkt der Moderne - im Wesentlichen also ein Vorgang innerhalb der entwickelten Welt und in Bereichen, in denen gesellschaftliche gegenüber staatlichen Akteuren schon über vergleichbare Kompetenzen verfügen. Es darf auch nicht vergessen werden, dass das Zugeständnis gesellschaftlicher Selbststeuerung oder der Mitentscheidung durch zivilgesellschaftliche Akteure ein staatliches Zugeständnis ist. ,Entstaatlichung' ist also ein Projekt, das die Aufgabe staatlicher Alleinbestimmung, die Zuweisung bestimmter Herrschaftsbereiche an gesellschaftliche Akteure und - in aller Regel - die staatliche Rahmensetzung, Regulierung, Überwachung und eventuell auch die Revision dieser Zuweisung beinhaltet.

So neu, wie ein Teil der Literatur glauben machen kann, ist dieser Entstaatlichungsvorgang allerdings nicht. Erstens ist darauf hinzuweisen, dass der Staat erst spät zum Herrschaftsmonopolisten wurde. Lange Zeit musste er seine Entscheidungsbefugnisse mit Kirche, Adel, Stammeshäuptlingen, Zünften und den Vertretungen freier Städte teilen. Das Monopol physischer Gewalt und der Steuererhebung konnte erst nach und nach durchgesetzt werden. Der Rechtsstaat heutiger Prägung, das allgemeine Wahlrecht und die staatliche Daseinsfürsorge sind neueren Datums (Leibfried und Zürn 2006). Selbst in den Zeiten der vollständigen Ausprägung des "demokratischen Interventionsstaates“ nach dem Zweiten Weltkrieg stellte der Staat nicht alle öffentlichen Leistungen allein zur Verfügung, sondern überließ sie - in unterschiedlichem Maße - der lose staatlich regulierten gesellschaftlichen Selbstverwaltung. Man denke etwa nur an die Aushandlung von Löhnen und Arbeitsbedingungen durch die Tarifpartner, die Administration der Sozialversicherung durch eigene Körperschaften, die private Trägerschaft von Bildungs- und Gesundheitsdiensten, die Selbstregulierung der Ärzte durch die Kammern und anderes mehr.

Allerdings hat sich die Entstaatlichung seit etwa drei Jahrzehnten beschleunigt. Das hat hauptsächlich mit der gesellschaftlichen und wirtschaftlichen Globalisierung und dem wachsenden Einfluss internationaler Organisationen und Regime sowie dem Wachsen einer internationalen Zivilgesellschaft zu tun, auf die im nächsten Abschnitt detaillierter eingegangen wird. Zum Teil ist sie aber auch Folge - und mit den genannten Vorgängen zusammenhängend - der abnehmenden Steuerungsfähigkeit hierarchisch organisierter, staatszentrierter Politik im nationalen Rahmen. Dazu nun einige Stichwörter:

Nach Jahrzehnten einer eher zufällig und vornehmlich von der Verfügbarkeit vorhandener Steuermittel geprägten Politik verlegten sich viele Regierungen ab Mitte der 1960er Jahre auf eine stärkere Planung mittel- und langfristiger Abläufe (beginnend mit einer aktiven Konjunkturpolitik). Sie legten sich entsprechende Planungsstäbe zu und versuchten politisch-gesellschaftliche Entwicklungen planmäßig zu steuern. 
Der damalige Planungsoptimismus machte recht bald einer gewissen Ernüchterung Platz, weil sich komplexe gesellschaftliche Prozesse nun einmal nicht feinsteuern lassen, die Planung durch nicht vorhersehbare Krisen aus dem Kurs geworfen wurde. Hoheitliche Planung kann zwar beschlossen werden, zu ihrer Umsetzung bedarf es aber erstens der Mitwirkung der Verwaltung auf allen Ebenen und der Akzeptanz durch die Planungssubjekte, also durch die Bevölkerung und der sie mit vertretenden Verbände, Vereinigungen und sozialen Bewegungen. Damit haperte es aber mit dem Aufkommen von Bürger- und Studentenbewegungen und dem Erstarken neuer, vor allem grüner Parteien, mehr und mehr.

Zweitens weiteten sich die staatlichen Aufgaben nach dem Zweiten Weltkrieg so stark aus, dass der Staatsapparat finanziell und organisatorisch an seine Grenzen stieß. Das betraf zunächst die staatlichen Produktions- und Versorgungsbetriebe, deren Leistungsfähigkeit stets in der Kritik gestanden hatte, sich aber in Zeiten schwindender Haushaltsmittel $\mathrm{zu}$ einer zunehmenden Belastung entwickelte. Sie wurden teilweise durch technologische Entwicklungen überrollt, die ihr Leistungsmonopol fragwürdig und überflüssig machten (z. B. Mobiltelefone, Satellitenfernsehen etc.). Kurzum, ab Ende der 1970er Jahre gab es eine massive Welle der Privatisierung staatlicher Unternehmen und Leistungen, die später auch auf weniger entwickelte Staaten überschwappte und - in Begleitung des Stellenabbaus - zu einem deutlichen Schrumpfen des staatlichen Wirtschaftssektors führte. Der Zusammenbruch der sozialistischen Staaten ab Ende der 1980er Jahre führte dann vollends zur Delegitimierung des staatswirtschaftlichen Modells. Die weiter anhaltende Haushaltskrise zwang die meisten Industriestaaten später noch mehr dazu, Leistungsbereiche an nicht-staatliche Träger abzugeben, gemeinsam zu verwalten oder Leistungen schlicht einzuschränken.

Drittens nahmen im Zuge der wirtschaftlichen Entwicklung und der starken Verbreiterung und Verbesserung des Bildungsangebots neue soziale (Mittel-) Schichten in den Industrieländern zu, die stärker an "postmateriellen“ Werten der Selbstverwirklichung, Bürgerbeteiligung und gesellschaftlichen/ökologischen Nachhaltigkeit interessiert waren als am bloßen Funktionieren politischer/wirtschaftlicher Abläufe sowie an den daraus bezogenen Einkünften. Neue soziale Bewegungen und grüne Parteien forderten eine stärker dezentrale, partizipatorische Entwicklung und hatten damit Erfolg.

Schließlich brach sich das staatliche Entscheidungs- und Herrschaftsmonopol an den stets komplexer werdenden Verhältnissen, die zu planen und regulieren sind und die Detailkenntnisse der Politiker und der Bürokratie schlicht überfordern würden, wenn sie nicht die Expertise anderer Akteure nutzen könnten. Auch das hat in nicht geringem Maße mit der wachsenden internationalen Politikverflechtung zu tun. 
Im Ergebnis gibt es heute kaum mehr einen Herrschaftsbereich, in dem private Akteure von der Durchführung öffentlicher Organisationsaufgaben prinzipiell ausgeschlossen sind. Es gibt mittlerweile selbst private Sicherheitsdienste, die Steuerschulden werden teilweise durch private Inkassounternehmen eingetrieben, die meisten staatlichen Dienste (Post, Bahn, Gas, Wasser, Elektrizität, Telefon) sind privatisiert oder zumindest der privaten Konkurrenz geöffnet worden, staatliche Beteiligungen in strategischen Industriesektoren und im Finanzsektor sind zurückgefahren oder aufgegeben worden, staatliche Sozialversicherungssysteme werden durch privat organisierte Zusatzversorgungen ergänzt. Der Vollständigkeit halber muss man unterstreichen, dass dieser Prozess nicht unumkehrbar ist (wie etwa die teilweise Übernahme illiquider Banken in staatliche Hand im Zuge der letzten internationalen Finanzkrise zeigt). Dieser Kurswechsel könnte notwendig werden, wenn der Markt und private Dienste offensichtlich versagen und/oder sich massive politische Widerstände gegen die fortgesetzte private Leistungserbringung einstellen. Damit wird deutlich, dass die so umrissene Governance, ebenso wie die weiter unten abzuhandelnde Global Governance ein politisches Projekt ist, das von staatlichen Instanzen gewollt und gefördert werden muss.

\subsection{Global Governance}

Die Attraktivität des Begriffs und des damit verbundenen Programms von Global Governance speisen sich zu nicht geringen Teilen aus der Vorstellung, dass sie geeignet sind, Politikfelder zu bearbeiten, die sich aus der zunehmenden wirtschaftlichen, sozialen, medialen und kulturellen Vernetzung der Welt ergeben. Diese Vorgänge werden seit einiger Zeit als Globalisierung beschrieben; mit ihr zusammen hängt die Zunahme grenzüberschreitender Transaktionen und Wirkungsketten mit günstigen oder abträglichen Folgen für jene Staaten, die nicht deren Verursacher sind. Global Governance ist damit im Kern als der Versuch zu kennzeichnen, in Abwesenheit eines regulierenden Weltstaates die durch die Zunahme und Vertiefung der grenzüberschreitenden Vorgänge entstehenden Probleme durch internationale Kooperation - zusammen mit zivilgesellschaftlichen und wirtschaftlichen Akteuren - und gemeinschaftlich ausgehandelte Verregelung einzufangen oder zumindest zu bändigen (vgl. etwa Messner und Nuscheler 1996; Beck 1997; Behrens 2005). Die Absicht, die angeblich oder tatsächlich entfesselten Kräfte des Weltmarktes zu regulieren und damit politische Autorität über die damit verbundenen Vorgänge auf höherer Ebene wiederherzustellen, stellt jedenfalls den Kern 
normativer Ansätze von Global Governance dar. Analytische Ansätze zielen dagegen vornehmlich darauf ab, diese Entwicklungen, ihre Triebkräfte und Dynamik zu beschreiben.

Da die angesprochene Globalisierung eine entscheidende Motivation für solche Überlegungen darstellt, soll zumindest skizziert werden, inwieweit diese tatsächlich neue Herausforderungen an politische Steuerung stellen. Dieser Versuch stößt auf die Schwierigkeit, dass zu diesem Thema eine nicht mehr überschaubare Flut an Veröffentlichungen vorgelegt wurde, ohne einen Konsens darüber zu fördern, (a) was genau unter Globalisierung zu verstehen ist, (b) welche gesellschaftlichen Bereiche sie umfasst, (c) wann der Ausgang des Globalisierungsprozesses anzusetzen ist, (d) welche Regionen/Sektoren dabei erfasst wurden und (e) inwieweit die staatliche Souveränität von diesem Vorgang untergraben wurde.

Unter Globalisierung wurde zunächst nur die Intensivierung des grenzüberschreitenden Austauschs von Gütern, Dienstleistungen, Kapital und - seltener - die Zunahme der internationalen Migration verstanden. Der Begriff wurde also ökonomisch verkürzt, später wurden die kulturelle Dimension, der Informationsaustausch, der Zuwachs internationaler Organisationen und Regime, die Ausbildung einer internationalen Zivilgesellschaft und die Zunahme globaler Risiken (etwa Erderwärmung, transnationaler Terrorismus etc.) hinzugefügt. Problematisch war aber auch, dass Globalisierung quantitativ an der Erhöhung der Außenhandelsquote (am Bruttoinlandsprodukt), des Anteils ausländischen Kapitals an den gesamten Investitionen, dem Anteil von Auslandspost und ähnlichem festgemacht wurde. Das macht deswegen nicht viel Sinn, weil es Handels-, Kapital- und Postverkehr ja schon lange gibt. Mit seiner bloßen Zunahme geschieht nicht wirklich Neues. Es ist auch nicht nachzuvollziehen, wie dadurch die Steuerungsfähigkeit der Nationalstaaten hätte untergraben werden können.

$\mathrm{Zu}$ Recht wurde darauf verwiesen, dass der Güter- und Kapitalaustausch schon kurz vor dem 1. Weltkrieg einen beachtlichen Umfang erreicht hatte, der - relativ - erst wieder in den 1990er Jahren übertroffen wurde. Auch der Versuch, Globalisierung mit Maßnahmen der Deregulierung gleichzusetzen, also der Senkung der Zollsätze oder der Beseitigung von Restriktionen des internationalen Kapitalverkehrs, führt nicht viel weiter. Auch diese Maßnahmen gab es schon früher, und sie müssen nicht zwangsläufig zu steigender internationaler Vernetzung führen. Sinn macht der Begriff Globalisierung nur, wenn damit etwas qualitativ Neues bezeichnet wird (ebenso Scholte 2000; Reinicke 1998). Zwei Aspekte scheinen diesbezüglich relevant zu sein: Erstens die massive Beschleunigung der Transaktionen, etwa die sekundenschnelle Übertragung von Nachrichten, Mitteilungen, Bestellungen und der ebenso schnelle Zugang bzw. Abzug von Kapital aus/in Volkswirtschaften mit der Folge zunehmender 
internationaler Konvergenz an den Aktien- und Anleihemärkten der Welt und entsprechend simultan auftretender Krisenerscheinungen. Zweitens zerlegen sich die Produktionsprozesse von Gütern und Dienstleistungen in einzelne Segmente nach global ausgeloteten Kostengesichtspunkten innerhalb eines global operierenden Unternehmens. Um ein simples Beispiel zu geben: In ein aufwändiges Endprodukt gehen heute Vorleistungen und Zwischengüter aus vielen Standorten in unterschiedlichen Staaten ein. Diese werden durch Investoren und Kreditinstitute weltweit finanziert. Forschung, Produktion, Absatz, Werbung werden am jeweils günstigsten Standort getätigt und Gewinne dort ausgewiesen, wo die Steuerlast und die staatlichen Leistungen in einem günstigen Verhältnis stehen.

Das ist wirklich neu und führt in der Tendenz dazu, die traditionellen wirtschaftspolitischen Steuerungsmittel des Nationalstaates - also Geld-, Fiskal- und Währungspolitik - teilweise außer Kraft zu setzen, weil sie mit Abzug von internationalem Kapital oder Abwanderung von Unternehmensteilen seitens privater Akteure beantwortet werden könnten. Diese Art von Globalisierung setzt möglicherweise den Sozialstaat unter Druck, wenn dieser den Standort durch hohe Lohnnebenkosten oder steuerfinanzierte Transfers belastet. Inwieweit das tatsächlich geschieht und in welcher Dimension, wird kontrovers beurteilt. Ähnlich erodiert mit der Verbreitung des Internets auch die informationelle Souveränität des Nationalstaates. Es zeichnet sich dadurch eine neue Weltöffentlichkeit $\mathrm{ab}$, die Ereignisse andernorts registriert, kommentiert und sich gegebenenfalls zu Gegenreaktionen organisiert (s.u.). Schließlich sind mit dem rascheren Wachstum der Weltwirtschaft und der engeren Verflechtung der Staaten die grenzüberschreitenden Risiken gestiegen (Klima, kriminelle Netzwerke, Terrorismus etc.).

Nicht alle Sektoren, Staaten und sozialen Gruppen sind gleichermaßen in die Globalisierung einbezogen. Viele Staaten sind vom Weltmarkt noch teilweise abgeschottet oder für transnational operierende Konzerne trotz wirtschaftspolitischer Öffnung als Standorte nicht interessant. Die Mehrzahl der Arbeitskräfte produziert für den lokalen Markt, der Löwenanteil der Investitionen wird aus nationalen Ersparnissen finanziert und auch eine vollständige Angleichung der nationalen Zins- und Kreditraten sowie der nationalen Steuersätze hat keinesfalls stattgefunden. Auch im Prozess der Globalisierung leisten sich die Staaten ein sehr unterschiedliches sozialpolitisches Engagement. Ferner setzt die Teilnahme an diesem Prozess voraus, dass die involvierten Personen und Gruppen über die nötigen Voraussetzungen verfügen (etwa einen eigenen oder zumindest leicht zugänglichen Internetanschluss oder in der internationalisierten Produktion verwertbare professionelle Fähigkeiten). Schließlich ist die Beteiligung an der internationalen Zivilgesellschaft sehr ungleich, und 
internationale Organisationen haben nur in wenigen Bereichen die Kompetenz, nationales Gesetz zu ersetzen oder gar zu brechen. Daher ziehen etliche Autoren den Begriff „Denationalisierung" gegenüber dem Begriff Globalisierung vor (Zürn 1998). Zuletzt muss erneut betont werden, dass Globalisierung, also die Öffnung der Märkte, das Zulassen von Arbeitsmigration und ausländischen Medien, ein von nationalen Regierungen erlaubtes oder sogar aktiv betriebenes Projekt darstellt, also nicht vom Himmel gefallen ist.

Zum nationalstaatlich geprägten Ursprungszustand zurückzukehren, fällt aber nicht leicht, weil dies erhebliche Einbußen verlangen würde. Daher wird das Programm einer Global Governance, also der Versuch, das Auseinanderfallen von nationalstaatlich begrenzter Regulierungsfähigkeit bei gleichzeitiger Zunahme grenzüberschreitender, globaler Interaktionen und Probleme durch internationale Regulierung (unter Beteiligung einer wie auch immer gearteten Zivilgesellschaft) zu kompensieren, auf der Tagesordnung bleiben. Unter Global Governance ist - zusammengefasst - also Folgendes zu verstehen:

1. ,Weltregieren' ohne einen Weltstaat, dem allein Steuerungs- und Kontrollfähigkeit zukäme, vielmehr horizontale (verschiedene Akteure auf gleicher Augenhöhe) wie vertikale (verschiedene Ebenen verschränkende) Formen der Koordination staatlicher, gemischter oder privater Akteure zum Zweck der Verregelung transnationaler Interaktionen, zur Harmonisierung politischer Interventionen, zur Umsetzung der vereinbarten Beschlüsse (inklusive eventueller Sanktionen gegenüber Abweichlern), der Überprüfung ihrer Wirksamkeit und ihrer gegebenenfalls nötigen Korrektur.

2. Ebenso wenig wie Governance meint Global Governance Regieren durch Kommando und Kontrolle vornehmlich oder gar ausschließlich. Das wäre allein schon deshalb unsinnig, weil wie auch immer getroffene internationale Vereinbarungen und Regelungen durch nationale staatliche oder private Akteure ratifiziert und umgesetzt werden müssen. Es kann also nur darum gehen, durch internationale Verhandlungen einen möglichst konsensfähigen Rahmen zu schaffen, der - bei normalerweise fehlenden harten Sanktionsinstrumenten gegenüber Abweichlern - freiwilligen Vollzug garantiert. Problemspezifische Netzwerke werden als die geeignetsten Werkzeuge zur Schaffung des für Vereinbarungen nötigen Sachverstands erachtet.

3. Global Governance stützt sich auf zahlreicher gewordene internationale Organisationen und internationale Regime - also auf problemspezifische, internationale Regelungen, die bestimmen, nach welchen Normen und Umsetzungsverfahren spezifische Probleme zu lösen sind - und mehr noch auf deren tiefergehenden, zuweilen schon suprastaatlichen Kompetenzen (Beispiele: Europäische Union, 
Welthandelsorganisation, internationaler Strafgerichtshof). Diese Organisationen treffen nicht nur wichtige allgemein-verbindliche Entscheidungen, sondern setzen diese auch mit eigenen Organisationsressourcen um, vor allem bei der Standardisierung und Zertifizierung von Produkten und Verfahren, z. T. auch bei der Zuteilung globaler Rechte (etwa der Domain-Namen durch ICANN) oder bei der Vergabe der ihnen anvertrauten Mittel (Genschel und Zangl 2008).

4. Bei Fehlen eines Weltparlaments, welches diese Vereinbarungen beschließen und legitimieren könnte, stellt sich das Problem der demokratischen Mitbestimmung in Institutionen von Global Governance. Der Verweis auf die Delegation der Mitbestimmung auf die gewählten Regierungen durch die Bürger vermag nicht wirklich zu überzeugen. Denn die Verantwortungskette zwischen Bürgern und internationalen Verhandlungsführern ist sehr lang, die Verhandlungsmaterie sehr komplex, und sie übersteigt das Verständnis der Mehrheit nationaler Abgeordneter deutlich. Da es auch den bestehenden internationalen Organisationen bisher nur mäßig gelungen ist, sich eine eigene Legitimationsbasis zu verschaffen, sehen viele Autoren in der Gestalt der internationalen Zivilgesellschaft eine Art Ersatzparlament, das eine Art Wachhundfunktion in Bezug auf Global Governance-Entscheidungen und ihre Umsetzung ausüben soll. Die Wahrnehmung der internationalen Zivilgesellschaft als Hoffnungsträger für ein stärker legitimiertes Weltregieren ergibt sich daraus, dass seit den 1980er Jahren die Zahl der international operierenden Nichtregierungsorganisationen (NGOs) mächtig zugenommen und ihre Vernetzung sich stark verdichtet hat. Ihren Aktivitäten ist es unter anderem zu verdanken, dass Themen wie die Wahrung der globalen Umweltbedingungen, der Schutz der Menschenrechte, der Kampf gegen Korruption etc. auf die internationale Agenda kamen (Behrens 2013). Die NGOs wurden überdies sukzessiv in Konsultativgremien internationaler Organisationen eingebunden. In etlichen internationalen Organisationen wurden nicht-staatlichen Akteuren mittlerweile sogar institutionalisierte Mitwirkungerechte eingeräumt (z. B. auch im Internet Governance Forum). Diese wurden ihnen nicht nur freiwillig zugestanden, sondern um Entscheidungen und ihrem Zustandekommen höhere Legitimität zu verleihen und die Expertise dieser Zivilgesellschaft zu nutzen (Rittberger u. a. 2010).

5. Bei der nun schon länger positiven Bewertung der entstehenden Zivilgesellschaft wird oft vergessen, dass zu dieser auch private Wirtschaftsunternehmen bzw. deren Vertreter gehören, die oder deren Verbände in etlichen Fällen durchaus transnationale wirtschaftliche Konflikte in Eigenregie, also ohne hoheitliche, staatliche Beteiligung, lösen. Ein Beispiel hierfür ist die lex mercatoria, die Regeln für die zwischenstaatliche Streitbeilegung für Privatunternehmen vorgibt. 
6. In Summe heißt dies: In etlichen Sachbereichen der Weltpolitik sind die Nationalstaaten heute nicht mehr die einzigen - mitunter auch nicht mehr die dominierenden Akteure. Freilich sind diese Staaten nach wie vor „nicht einfach Werkzeuge unsichtbarer weltgeschichtlicher Kräfte" (Ebd.). Sie sind nach wie vor selbst Architekten der internationalen Ordnung und damit auch Urheber, treibende Kräfte und Gestalter der Globalisierung. Auch sind sie durchaus in der Lage, den Prozess der stärkeren Delegation von Mitentscheidungsrechten internationaler Organisationen und der Zivilgesellschaft zurückzudrehen, wie das unilaterale Verhalten der USA zu Zeiten des vormaligen Präsidenten Bush hinlänglich demonstriert hat. Man kann sinnvollerweise unterscheiden Governance by, with and without governments, je nachdem ob Staaten ohne internationale Organisationen und private Akteure allgemeingültige Entscheidungen fällen, mit diesen oder Letztere alleine.

Man kann sich dem Projekt der Global Governance nicht nur bestätigend, sondern auch kritisch nähern. Das tun allerdings nur wenige Vertreter. Sie sehen in ihr den ideologischen Überbau einer neoliberalen Konzeption weltwirtschaftlicher Zusammenarbeit, die sich nicht um die kritische Auseinandersetzung der internationalen Herrschaftsstrukturen bemüht. Auch müssten die Ursachen geklärt werden, welche die gesellschaftlichen Probleme hervorgebracht haben, die nun angeblich einer Regulierung bedürfen. Vielmehr werde einem technokratischen Politikverständnis gehuldigt, das die ungleiche interne und internationale Machtverteilung nicht oder zu wenig thematisiere. Die Zivilgesellschaft diene im Wesentlichen der Entlastung staatlicher Politik, und sie werde von Vereinigungen und NGOs aus den etablierten Industriestaaten dominiert. Global Governance sei also „die allzu versöhnlerische Utopie einer Weltgesellschaft, in der alle widerstreitenden Interessen unter Aufsicht der Staaten zu allseitiger Zufriedenheit aufgelöst werden“ (Brand u. a. 2000, 157). Diese These ist nicht ganz falsch. 